\title{
RAYLEIGH TAYLOR INSTABILITIES IN THE INTERSTELLAR MEDIUM
}

\author{
Marc Lachièze-Rey \\ Section d'Astrophysique \\ Centre d'Etudes Nucléaires de Saclay, France
}

Parker $(1966,1969)$ proposed that the interstellar medium could be subjected to instabilities, able to destroy its structure in a time shorter than the galactic evolution time. He emitted the idea that this phenomenon could lead to halo formation, escape of magnetic field and cosmic rays from the galaxy, via some sort of interstellar "bubbles", and also to cloud formation. He showed then that if the interstellar medium was well represented by a simple horizontal stratified equilibrium model, perturbations would effectively grow and destroy the structure.

The equilibrium state whose stability he studied was however very schematic. On the other hand, his work was discussed and criticized by various authors. We tried to have a new approach to the problem and to answer the questions which remained open.

In this perspective, we built a tool to test the stability of any 2 dimensionnal equilibrium configurations able to represent the interstellar medium (Asseo et al.1978,1980): following the idea of Zweibel and Kulsrud (1975), we adapted the energy principle used by Bernstein et al.(1958) to test hydromagnetic configurations. We developed it for the composite interstellar medium made of interstellar gas and cosmic rays gas, immersed in the galactic magnetic field and in the gravitational field of the galactic stars.

1) For horizontal configurations (where the magnetic field lines remain parallel to the galactic plane), we derived a global instability criterion (Lachièze-Rey et al.1980): it concerns all the types of perturbation which can apply to the configuration. This criterion shows us that all the configuration proposed by Badhwar and Stephens (1977) to depict the interstellar medium are unstable. Calculation of the growth rates ensures us that perturbations have enough time to grow. 
2 ) For non horizontal configurations, we were not able to derive a necessary and sufficient instability criterion. In the case $\gamma=1$ and $\Gamma=O(r$ and $I$ ' are the polytropic indexes of the interstellar and cosmic ray gases respectively) we give however sufficient instability criteria, corresponding to special types of perturbation. They permit us to exhibit the destabilizing effects of the curvature of the magnetic field lines and of the cosmic rays (Asseo et al.1978,1980). We apply them to a family of possible equilibrium configurations, introduced by Mouschovias (1974). These configurations, related to horizontal states by a flux-conserving possible evolution, were proposed to be the result of the development of instabilities from horizontal states. We show that all are also unstable, including the one calculated numerically by Mouschovias. We give also the value of growth's rates.

3 ) We also studied the effect of hydromagnetic turbulence, as suggested by Zweibel and Kulsrud (1975), on the stability of horizontal configurations: the short wavelengths are completely stabilized, the longer ones $(\sim 100 \mathrm{pc}$ ) only partially (Lachièze-Rey et al.1980). We also discuss the values of $\gamma$ and $\Gamma$ and give some estimates of the influence of $\gamma \neq 1$ and $\Gamma \neq 0$ on the stability (Lachièze-Rey and Pellat 1980).

In conclusion, we showed that all the configurations already proposed to depict interstellar medium are unstable, and therefore not able to survive on the galactic time scale, although it is not clear if the unstabilities only generate small scale turbulence or lead to the formation of big scale structure, the escape of cosmic rays and magnetic field and the concentration of the gas near the plane.

\section{REFERENCES}

Asseo,E. ,Cesarsky,C.J. ,Lachièze-Rey,M. ,and Pellat,R., 1978, Ap.J.Letters, $\underline{225}$, L21-25

Asseo,E.,Cesarsky,C.J.,Lachièze-Rey,M., and Pellat,R., 1980, Ap.J. ( 1 May 1980)

Badhwar,G.D. and Stephens,S.A., 1977, Ap.J., 212, 494

Bernstein,I.B.,Frieman,E.A.,Kruskal,M.D., and Kulsrud, R.M., 1958, Proc. Roy.Soc.London, $\underline{A} 244,17$

Lachièze-Rey,M.,Asseo,E.,Cesarsky,C.J., and Pellat R., 1980, Ap.J. (15 May 1980)

Lachièze-Rey,M., Pellat,R., 1980 (in preparation)

Mouschovias,T.Ch., 1974, Ap.J., 192, 37

Parker,E.N., 1966, Ap.J., 145, 811

Parker,E.N., 1969, Space Sci.Rev., 9, 651

Zweibel,E.G., and Kulsrud,R.M., 1975, Ap.J., 201, 63 